\title{
Comparação da eficiência de dois métodos de coleta de peixes em igarapés de terra firme da Amazônia Central
}

\author{
Odirlene Marinho RIBEIRO ${ }^{1} \&$ Jansen ZUANON $^{1}$
}

\section{RESUMO}

Em estudos sobre distribuição espacial, alimentação e estrutura de comunidades de espécies de peixes de pequeno porte em igarapés, são aplicados alguns métodos de coleta específicos para esses ambientes. Este trabalho teve como objetivo principal comparar dois métodos de coleta, um ativo (puçá e peneira) e outro passivo (armadilhas). A coleta com puçá foi mais eficiente, tanto em número de espécies registradas, quanto em número de indivíduos capturados. Houve uma taxa de escape aparentemente elevada nas armadilhas, assim como a predação de parte dos peixes capturados por jejus (Erythrinus erythrinus) dentro das armadilhas, o que pode ter contribuído para a menor eficiência desse método. A baixa similaridade entre os pares de amostras coletadas com os dois métodos em cada igarapé indica a necessidade de utilizar diferentes métodos de coleta para assegurar a efetividade das amostragens. Entretanto, o menor impacto ao ambiente causado com o uso de armadilhas pode ser especialmente conveniente para estudos em Unidades de Conservação, desde que o esforço de coleta seja adequadamente dimensionado.

\section{PALAVRAS-CHAVE}

Ictiofauna, riqueza de espécies, riachos, armadilha, puçá.

\section{Comparison of the efficiency of two fish collecting methods in small forest streams in CentralAmazon}

\begin{abstract}
Studies of spatial distribution, feeding habits and community structure of small size fish species in forest streams usually employ some specific collecting methods related to the size and structural characteristics of these aquatic environments. This study aimed to compare the efficiency of two fishing methods, one active (hand net and sieve) and one passive (minnow traps). Collecting with hand nets yielded a higher number of fish species and individuals. There was apparently a high escape rate in the minnow traps, as well as some losses of collected specimens by predation inside the traps by the carnivorous Erythrinus erythrinus, that may have resulted in the lesser efficiency of the passive fishing method. The low similarity values observed in the samples obtained with the two fishing methods at each stream indicate that is necessary to combine different sampling procedures to ensure its effectiveness. Nevertheless, the lower habitat disturbance resulting with the use of minnow traps may be especially useful for studies developed in protected areas, with a proper adjustment of the sampling effort.
\end{abstract}

\section{KEY WORDS}

Ichthyofauna, species richness, creeks, minnow traps, hand nets. 
A regiāo Amazônica apresenta a maior bacia hidrográfica do mundo, formada pelo rio Amazonas e um incontável número de outros rios e pequenos riachos (igarapés) (Lowe-McConnell, 1999). Com exceção de boa parte dos rios maiores de águas brancas, cuja origem é andina, a maioria dos rios amazônicos resultam da junção de pequenos igarapés que drenam a floresta (Walker,1991; 1995).

Apesar da Amazônia abrigar a mais rica ictiofauna de água doce do planeta, seus igarapés são sistemas aquáticos com uma produção biológica baixa (Goulding et al., 1988), que dependem da floresta ripária como principal fonte de energia para sustentação da cadeia trófica (Walker, 1991; Lowe-McConnel, 1999). Estudos sobre distribuição espacial, alimentação e estrutura de comunidades de peixes em pequenos igarapés vêm sendo realizados na região amazônica (e.g., Silva, 1992; Sabino \& Zuanon, 1998; Bührnheim \& Cox-Fernandes, 2001). Na realização desses estudos, as metodologias de coleta aplicadas incluem métodos passivos (redes e armadilhas, como covos e "fike-nets") e ativos (redes de mão, puçás, peneiras, observação durante mergulho) (Thompson et al., 1998). Entretanto, pouco se sabe sobre a eficiência diferencial desses métodos e seus possíveis impactos sobre os resultados obtidos.

A coleta passiva consiste na captura de peixes utilizando apetrechos que não são movidos ativamente pelo coletor, produzindo supostamente uma menor perturbação no ambiente. Porém, a coleta passiva parece ser bastante seletiva quanto às espécies e ao tamanho dos peixes capturados (Uieda \& Castro, 1999). A coleta ativa, por outro lado, consiste na captura de peixes com uso de instrumentos que perturbam o ambiente, tanto pela movimentação dos coletores quanto pelas alteraçôes provocadas na estrutura dos microhábitats (e. g., bancos de serapilheira submersa, troncos). Além disso, as capturas por esse método podem ser altamente dependentes da habilidade do coletor (Uieda \& Castro, 1999).

Quando se considera a intensidade dos dois tipos de impactos de coleta sobre as populaçôes naturais, especialmente em áreas de unidades de conservação ambiental, seria recomendável o uso de métodos de coleta passivos. Por outro lado, quando há necessidade de realizar levantamentos ecológicos ou faunísticos rápidos, os métodos ativos, via de regra, são escolhidos pela sua suposta maior eficiência por unidade de tempo.

O presente trabalho teve por objetivo comparar a eficiência de duas metodologias de coleta (armadilhas do tipo covo e coleta ativa com puçás e peneiras) em igarapés de terra firme da Amazônia Central, em relação à riqueza, composição de espécies e abundância das capturas.

Os trabalhos de campo foram realizados no período de 28 de julho a 01 de agosto de 2003, em oito igarapés de primeira ordem da Reserva do Km 41, do Projeto de Dinâmica Biológica de Fragmentos Florestais (PDBFF-INPA/Smithsonian Institution). A reserva fica localizada a $80 \mathrm{~km}$ a Noroeste da cidade de Manaus $\left(2^{\circ} 24^{\prime} 26^{\prime \prime}-2^{\circ} 25^{\prime} 31^{\prime \prime} S, 59^{\circ} 43^{\prime} 40^{\prime \prime}\right.$ $59^{\circ} 45^{\prime} 50^{\prime \prime} \mathrm{O}$ ) (Tabela 1), com altitude variando de 50 a $125 \mathrm{~m}$ sobre o nível do mar. A reserva apresenta um sistema de trilhas que possibilitou a escolha prévia dos igarapés estudados, baseada nas características de tamanho e estrutura dos mesmos, de forma a permitir uma comparação adequada dos resultados.

As coletas foram realizadas em dois trechos de $20 \mathrm{~m}$ por igarapé, bloqueados com tela plástica de mosquiteiro (malha $=1$ $\mathrm{mm}^{2}$ ) e distanciados entre si em cerca de $5 \mathrm{~m}$. Para minimizar os possíveis efeitos de características ambientais locais sobre os resultados das coletas com cada método, sempre foram escolhidos trechos de igarapé com feições semelhantes em termos de estrutura morfológica do canal e complexidade estrutural do ambiente aquático (diversidade de microhabitats). A coleta ativa sempre foi realizada no trecho localizado a jusante, de forma a evitar possíveis interferências diretas da perturbação produzida pela coleta sobre as capturas com as armadilhas. Após a delimitação da área, foram tomadas três medidas de largura do igarapé (medida com trena, em cm) e três medidas de velocidade (por meio da cronometragem do tempo de deslocamento de um objeto flutuante por uma distância conhecida). Apesar de todos os igarapés amostrados serem de primeira ordem (Petts, 1994), a largura varia razoavelmente e constitui um bom descritor do tamanho desses corpos d'água (JZ, obs. pess.). A profundidade máxima do canal foi medida com uso de um bastão graduado, no ponto mediano do trecho de $20 \mathrm{~m}$ do igarapé. Tomando como base a área de uma hemi-elipse, foi possível estimar a vazão de cada trecho de igarapé pela fórmula: $Q=A * V m$, onde $Q$ éa vazão; A é a área da secção transversal do igarapé; e Vm é a velocidade média da correnteza.

No método de coleta passiva, foram utilizadas 10 armadilhas tipo covo por igarapé, feitas de garrafa "pet" com 2 litros de volume (Figura 1), que permaneceram armadas por um período de 24h. Cada armadilha continha isca de sardinha em conserva acondicionada em um frasco plástico perfurado (volume $=36 \mathrm{ml}$ ). As armadilhas foram distribuídas ao longo do trecho de $20 \mathrm{~m}$ de igarapé, espaçadas em cerca de $2 \mathrm{~m}$ entre si e instaladas junto ao fundo de áreas marginais, sempre com a abertura voltada para jusante. No método ativo foi utilizado puçá (= rapiché, $0,30 \mathrm{x}$

Tabela 1 - Igarapés amostrados na área da Reserva do Km 41 (INPA/ PDBFF) com suas respectivas coordenadas geográficas.

\begin{tabular}{|c|c|}
\hline Igarapés & Coordenadas \\
\hline 1 & $02^{\circ} 26^{\prime} 59,7^{\prime \prime}$; $59^{\circ} 46^{\prime} 08,4^{\prime \prime} 0$ \\
\hline 2 & $02^{\circ} 26^{\prime} 55,1^{\prime \prime S} ; 59^{\circ} 46^{\prime} 18,5^{\prime \prime} 0$ \\
\hline 3 & $02^{\circ} 26^{\prime} 51,4^{\prime \prime}$; $59^{\circ} 46^{\prime} 05,2^{\prime \prime} 0$ \\
\hline 4 & $02^{\circ} 26^{\prime} 32,1^{\prime \prime S} ; 59^{\circ} 47^{\prime} 18,2^{\prime \prime} 0$ \\
\hline 5 & $02^{\circ} 26^{\prime} 37,6^{\prime \prime}$; $59^{\circ} 46^{\prime} 40,4^{\prime \prime} 0$ \\
\hline 6 & $02^{\circ} 26^{\prime} 36,7^{\prime \prime} \mathrm{S} ; 59^{\circ} 46^{\prime} 44,8^{\prime \prime} 0$ \\
\hline 7 & $02^{\circ} 26^{\prime} 13,7^{\prime \prime}$; $59^{\circ} 46^{\prime 2} 26,4^{\prime \prime} 0$ \\
\hline 8 & $02^{\circ} 26^{\prime} 02,4^{\prime \prime} S ; 59^{\circ} 46^{\prime} 32,5^{\prime \prime} 0$ \\
\hline
\end{tabular}


$0,25 \mathrm{~m}$, malha $=1 \mathrm{~mm}^{2}$ ) e peneira de malha metálica de arame (diâmetro $=0,60 \mathrm{~m}$, malha $=3 \mathrm{~mm}$ ), tomando o cuidado de amostrar exaustivamente os diferentes ambientes encontrados (serapilheira, raízes, areia, barrancos). Em média, as coletas ativas duraram cerca de $1 \mathrm{~h}$ por trecho de igarapé, que foi considerado esgotado quando após $5 \mathrm{~min}$ de coleta não se capturava nenhum indivíduo adicional. Os peixes coletados com ambos os métodos foram colocados em bandejas plásticas, identificados e contados no próprio local e devolvidos ao igarapé no final da amostragem.

Após a aplicação dos dois métodos de coleta, surgiu a dúvida de que as armadilhas do tipo covo poderiam estar sofrendo uma alta taxa de escape de peixes, em função do longo intervalo de tempo (24h) entre a instalação e a despesca. Assim, decidiu-se realizar um experimento de campo com o objetivo de averiguar se os escapes realmente ocorriam e com qual freqüência. $\mathrm{O}$ experimento foi realizado durante o período matutino e durou cinco horas. Em um trecho de $40 \mathrm{~m}$ foram colocadas 20 armadilhas regularmente espaçadas. A cada hora todas as armadilhas eram revistadas, anotando-se as espécies e o número de indivíduos capturados. Em função da alta transparência da água do igarapé, essas revisōes foram realizadas visualmente, sem que os peixes fossem retirados das armadilhas. Quatro armadilhas foram removidas aleatoriamente a cada hora, como forma de medir as capturas efetivas por período. A taxa de escape foi medida pela diferença de captura em cada armadilha em horários subseqüentes.

Os valores de riqueza e abundância obtidos com as duas metodologias (apresentados na forma de média \pm desvio-padrão) foram comparados por meio de uma ANOVA (Zar, 1999). As relaçōes entre as capturas (abundância e riqueza de espécies) e características estruturais dos igarapés (largura e vazão) foram analisadas por meio de uma Análise de Covariância (ANCOVA). Uma regressão linear simples foi empregada para analisar a relação entre a riqueza de espécies registradas com os dois métodos de coleta combinados e a abundância de exemplares coletados em cada igarapé. Também foi avaliada a similaridade da composição de espécies registradas com cada método de coleta, com uso do índice de Jaccard (Krebs, 1999).

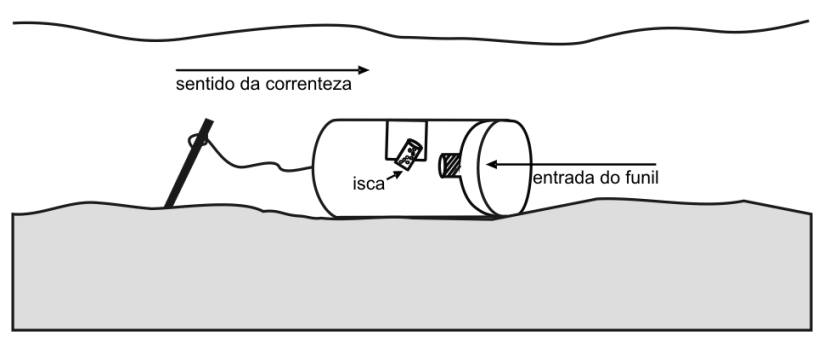

Figura 1 - Armadilha tipo covo (método passivo) utilizada para coleta de peixes em igarapés de terra firme.
Nos oito igarapés estudados foram registradas 17 espécies, em um total de 302 peixes. A ordem mais rica foi Characiformes com seis espécies, seguida por Siluriformes e Perciformes com quatro espécies cada; as ordens Gymnotiformes e Cyprinodontiformes apresentaram duas e uma espécies, respectivamente. A família mais rica foi Cichlidae, com quatro espécies (Tabela 2). $\mathrm{O}$ método ativo resultou em uma maior captura total (205 vs. 97 exemplares) e um maior número de espécies registradas (16 vs. 9). A média de capturas com o método ativo foi maior (25,6 peixes) do que com o passivo (12 peixes) $(\mathrm{F}=9,997 ; \mathrm{p}=0,007 ; \mathrm{n}=8)$. A riqueza de espécies também foi maior nas coletas com o método ativo $(\mathrm{F}=5,306 ; \mathrm{p}=0,038 ; \mathrm{n}=$ 8) (Figura 2).

Tanto a riqueza quanto a abundância não apresentaram relação com a largura (ANCOVA; riqueza: $\mathrm{F}=3,116 ; \mathrm{p}=0,101$; abundância: $\mathrm{F}=0,759 ; \mathrm{p}=0,399)$ nem com a vazão (ANCOVA; riqueza: $\mathrm{F}=1,086 ; \mathrm{p}=0,320$; abundância: $\mathrm{F}=0,093 ; \mathrm{p}=0,766$ ) dos igarapés, para os dois métodos de coleta utilizados. No
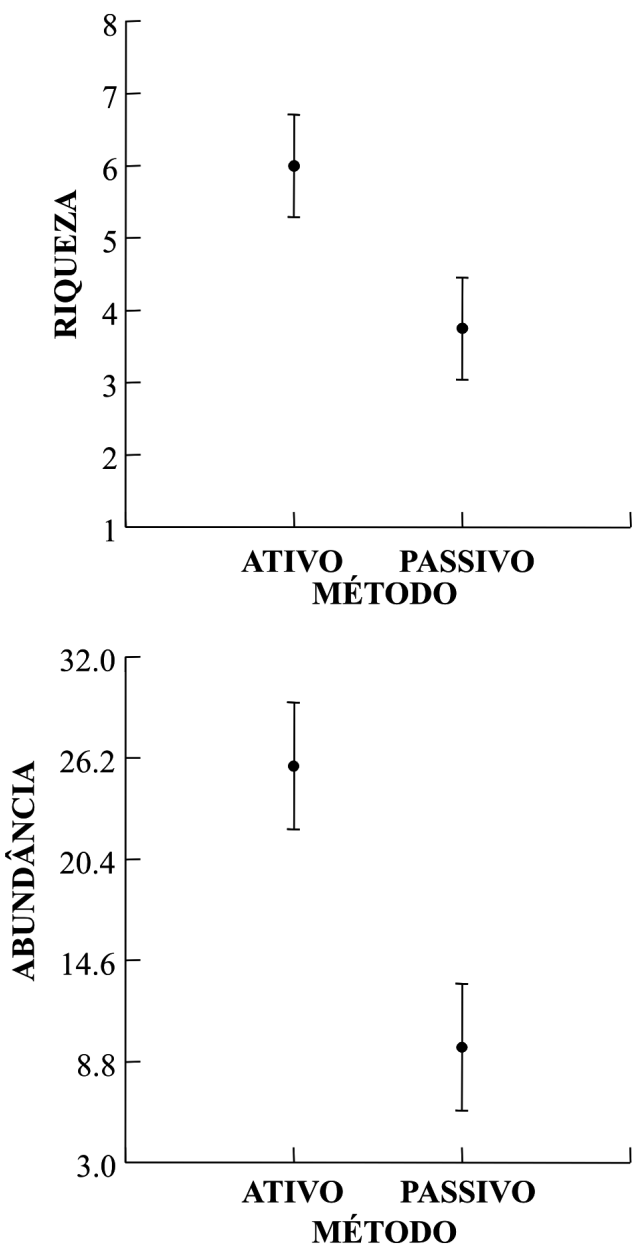

Figura 2 - Valores de riqueza de espécies e abundância de exemplares (média $\pm \mathrm{dp}$ ) coletados por meio de dois métodos de coleta (ativo = puçá; passivo $=$ armadilha), em trechos de $20 \mathrm{~m}$ de oito igarapés de terra firme. 
Tabela 2 - Lista de espécies capturadas em oito igarapés da Reserva do $\mathrm{Km}$ 41 (INPA/PDBFF) com uso de dois métodos de coleta (ativo = puçá; passivo $=$ armadilha).

\begin{tabular}{|c|c|c|c|}
\hline Espécie & Ativo & Passivo & Total \\
\hline \multicolumn{4}{|l|}{ CHARACIFORMES } \\
\hline \multicolumn{4}{|l|}{ Characidae } \\
\hline Hemigrammus cf. pretoensis & 30 & 6 & 36 \\
\hline Hyphessobrycon cf. agulha & 10 & 0 & 16 \\
\hline \multicolumn{4}{|l|}{ Crenuchidae } \\
\hline Microcharacidium sp. & 1 & 0 & 1 \\
\hline \multicolumn{4}{|l|}{ Lebiasinidae } \\
\hline Nannostomus marginatus & 4 & 5 & 9 \\
\hline Pyrrhulina cf. brevis & 105 & 64 & 169 \\
\hline \multicolumn{4}{|l|}{ Erythrinidae } \\
\hline Erythrinus erythrinus & 7 & 9 & 16 \\
\hline \multicolumn{4}{|l|}{ SILURIFORMES } \\
\hline \multicolumn{4}{|l|}{ Cetopsidae } \\
\hline Helogenes marmoratus & 11 & 0 & 11 \\
\hline \multicolumn{4}{|l|}{ Heptapteridae } \\
\hline Nemuroglanis sp. & 1 & 0 & 1 \\
\hline \multicolumn{4}{|l|}{ Trichomycteridae } \\
\hline Ituglanis cf. amazonicus & 2 & 0 & 2 \\
\hline \multicolumn{4}{|l|}{ Callichthyidae } \\
\hline Callichthys callichthys & 1 & 0 & 1 \\
\hline \multicolumn{4}{|l|}{ GYMNOTIFORMES } \\
\hline \multicolumn{4}{|l|}{ Gymnotidae } \\
\hline Gymnotus cf. pedanopterus & 2 & 0 & 2 \\
\hline Gymnotus anguillaris & 2 & 0 & 2 \\
\hline \multicolumn{4}{|l|}{ PERCIFORMES } \\
\hline \multicolumn{4}{|l|}{ Cichlidae } \\
\hline Aequidens pallidus & 2 & 4 & 6 \\
\hline Apistogramma aff. steindachneri & 1 & 3 & 4 \\
\hline Apistogramma sp. & 4 & 2 & 6 \\
\hline Crenicichla inpa & 0 & 1 & 1 \\
\hline \multicolumn{4}{|l|}{ CYPRINIFORMES } \\
\hline \multicolumn{4}{|l|}{ Rivulidae } \\
\hline Rivulus compressus & 22 & 3 & 25 \\
\hline Total & 205 & 97 & 302 \\
\hline
\end{tabular}

entanto, quando reunimos os resultados obtidos com os dois métodos, verificamos uma variação significativa da riqueza em função da abundância $\left(R^{2}=0,389 ; p=0,01 ; n=16\right)$ (Figura 3).

Houve similaridade geral de 0,65 na composição de espécies registradas com os métodos passivo e ativo. A similaridade média entre as capturas com os dois métodos em cada igarapé foi muito mais baixa $(0,27 \pm 0,18)$. O valor médio de similaridade entre igarapés amostrados com o método ativo foi de $0,41 \pm 0,17$ e

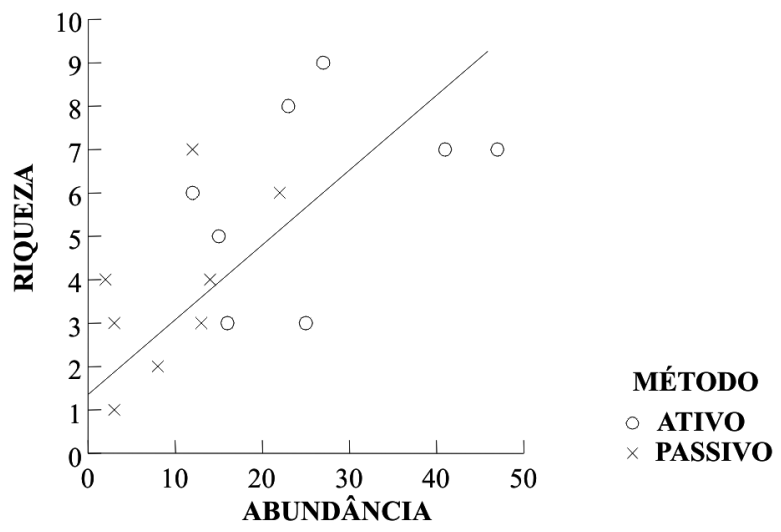

Figura 3 - Relação entre riqueza de espécies e abundância de peixes para os dois métodos de coleta combinados.

com o método passivo foi de $0,35 \pm 0,20$. Quanto à taxa de escapes de peixes, nas 40 vistorias sucessivas realizadas detectouse a ocorrência de escapes em 11 ocasiôes (27\%), representando uma perda potencial de 21 peixes.

O número de espécies encontradas nos oito igarapés amostrados neste estudo (17) foi semelhante ao registrado nos 12 pontos estudados por Araújo-Lima et al. (2000) nessa mesma área, que foi de 20 espécies. Isso indica que os dois métodos de coleta utilizados neste estudo apresentam eficiências comparáveis para amostragens em igarapés de primeira ordem. Araújo-Lima et al. (2000) encontraram uma relação significativa entre a abundância de peixes e o número de microhabitats, e não com a largura ou ordem do igarapé. Esses autores concluíram que amostragens em igarapés de primeira e segunda ordem seriam redundantes, em função da alta similaridade (efeito aditivo) na composição de espécies nesses ambientes. Um fator que pode explicar a aparente ausência de relação entre as capturas e as características estruturais dos igarapés no presente estudo é que os métodos de captura não são igualmente eficientes para igarapés de diferentes dimensōes. Características do habitat como profundidade, correnteza, tipo de substrato e transparência afetam a seletividade dos instrumentos de coleta (Uieda \& Castro, 1999), o que poderia estar comprometendo as capturas de certas espécies.

A diferença significativa na eficiência dos dois métodos de captura, tanto para riqueza quanto para abundância, indica que apesar de causar menos impacto, o método passivo é menos eficiente, o que, de acordo com o experimento realizado, foi reflexo de elevadas taxas de escape nas armadilhas. Talvez um redimensionamento da estrutura do covo, especialmente no funil de entrada, pudesse reduzir as taxas de escape. Em contraste, a captura de mais peixes com o método ativo resulta em uma maior probabilidade de registrar espécies pouco abundantes, raras ou que não são atraídas pelas iscas utilizadas. 
Durante as coletas regulares do experimento, também foi registrada a entrada de jejus (E. erythrinus) nas armadilhas. Aparentemente, a captura de jejus não acontecia em função da atração exercida pelas iscas, mas sim para comer os peixes menores aprisionados nos covos, o que também pode ter contribuído para reduzir o número de indivíduos capturados por esse método. A realização de vistorias mais freqüentes, com revisões de seis em seis horas por exemplo, provavelmente reduziria esse "ruído" nas amostragens, e possibilitaria a captura de indivíduos de acordo com o seu período de atividade (diurno ou noturno). No entanto, a praticidade de posicionar as armadilhas e retirá-las apenas no dia seguinte seria perdida, diminuindo a diferença de esforço em relação à captura ativa. Por outro lado, o método de coleta ativa pode gerar perturbaçōes na estrutura dos microhabitats, o que pode ser indesejável em situaçôes onde o ambiente ou a(s) espécie(s) encontram-se em condições muito vulneráveis, ou dentro de unidades de conservação de uso restrito.

Quanto à similaridade geral de 0,65 observada nas capturas, o método ativo permitiu a coleta de alguns peixes de hábitos criptobióticos, como: Callichthys callichthys, Helogenes marmoratus, Microcharacidium sp., Ituglanis cf. amazonicus, Gymnotus cf. pedanopterus e G. anguilaris, ausentes nas captura com o método passivo. Dentre as espécies capturadas com o método passivo, apenas uma, Crenicichla inpa, não ocorreu no método ativo, o que pode ser decorrência de uma baixa abundância dessa espécie nos igarapés. No entanto, os baixos valores de similaridade observados entre as amostras obtidas com a utilização de armadilhas indicam que para amostrar rápida e eficientemente os pequenos igarapés de terra firme, seria preciso conjugar os dois métodos ou aumentar o esforço de coleta. Comparações de métodos de pesca realizadas em diversos tipos de ambientes aquáticos têm demonstrado que os melhores resultados (tanto em termos de inventário ictiofaunístico, quanto de estimativas de abundância) são obtidos com uso de métodos pouco seletivos, como a rotenona (e. g., Dibble, 1991) ou a pesca elétrica (e. g., Pugh \& Schramm, 1998). Por outro lado, a comparação de métodos mais simples (e necesariamente mais seletivos) geralmente resulta na conclusão de que tais métodos produzem resultados complementares, e deveriam ser utilizados em conjunto (e. g., Goldstein, 1978). Os resultados obtidos no presente estudo também apontam para a necessidade de utilizar diferentes métodos de coleta como forma de garantir a efetividade das amostragens, considerando ainda as limitaçōes impostas pelo ambiente, o status de conservação da área a ser amostrada e a natureza do trabalho que se pretende desenvolver.

\section{AGRADECIMENTOS}

Ao PDBFF - INPA/Smithsonian Institution pela oportunidade de participar do curso de campo "Ecologia da Floresta Amazônica", durante o qual foram realizadas as coletas; aos companheiros L. Montag, A. Mortati, O. Pereira eA. Midori, pelo auxílio em campo. Esta é a contribuição \# 464 do PDBFF, e \# 04 do Projeto Ygarapés.

\section{BIBLIOGRAFIA CITADA}

Araújo-Lima, C. A. R. M; Jiménez, L. F; Oliveira, R. S.; Eterovick, P. C.; U. Mendoza, U.; Jerozolimnki, A. 2000. Relação entre o número de espécies de peixes, complexidade do habitat e ordem do riacho nas cabeceiras de um tributário do rio Urubu, Amazônia Central. Acta Limnologica Brasiliensia, 11(2): 127-135.

Bührheim, C. M.; Cox-Fernandes, C. 2001. Low seasonal variation of fish assemblages in Amazonian rain Forest streams. Ichthyological Exploration of Freshwaters, 12(1): 65-78.

Dibble, E. D. 1991. A comparation of diving and rotenone methods for determining relative abundance of fish. Transactions of the American Fisheries Society, 120: 663-666.

Goldstein, R. M. 1978. Quantitative comparison of seining and underwater observation for stream fishery surveys. The Progressive Fish-Culturist, 40: 108-111.

Goulding, M.; Carvalho, M. L.; Ferreira, E. G. 1988. Rio Negro, rich life in poor water. SPB Academic, The Hague, Netherlands. 200pp.

Krebs, C. J. 1999. Ecological Methodology. 2nd Ed., New York, USA. 619pp.

Lowe-McConnel, R. H. 1999. Estudos ecológicos de comunidades de peixes tropicais. Edusp, São Paulo. 535pp.

Petts, G. E. 1994. Rivers: Dynamic components of catchment ecosystems. In: Calow, P.; Petts, G. E. (Eds.). The river handbook. Vol. 2, Blackwell Scientific, Oxford. p. 3-32

Pugh, L. L.; Schramm, H. L. 1998. Comparation of electrofishing and hoopnetting in lotic habitats of the Lower Mississipi River. North American Journal of Fisheries Management, 18: 649-656.

Sabino, J.; Zuanon. J. 1998. A stream fish assemblage in Central Amazonia: distribution, activity patterns and feeding behavior. Ichthyological Exploration of Freshwaters, 8(3): 201-210.

Silva, C. P. D. 1992. Influência das modificaçôes ambientais sobre a comunidade de peixes de um igarapé da cidade de Manaus (Amazonas). Dissertação de Mestrado. INPA, Manaus. 112pp.

Thompson, W. L., White, G. C.; Gowan, C. 1998. Monitoring vertebrates populations. Academic Press, San Diego, CA, USA. 365pp.

Uieda, V. S.; Castro, R. M. C. 1999. Coleta e fixação de peixes de riachos. In: Caramaschi, E. P.; Mazzoni, R.; Peres-Neto, P. R. (Eds.). Ecologia de Peixes de Riachos, Série Oecologia Brasiliensis. Vol. VI. PPGE-UFRJ, Rio de Janeiro, Brasil. p. 1-22. 


\section{ACTA

Walker, I. 1991. Algumas considerações sobre um programa de zoneamento da Amazônia. In: Val, A. L.; Figliuolo, R.; Feldberg, E. (Eds.). Bases Cientificas para Estratégias de Preservação e Desenvolvimento da Amazônia. Vol. 1. INPA, Manaus. p. 37-46.

Walker, I. 1995. Amazonian streams and small rivers. In: Tundisi, J. G.; Bicudo, C. E. M.; Matsumura-Tundisi, T. (Eds.). Limnology in Brazil. Sociedade Brasileira de Limnologia, Academia Brasileira de Ciências, Rio de Janeiro, RJ. p. 167-193.
Zar, J. H. 1999. Biostatistical Analysis. $4^{\text {th }}$ Ed., Prentice Hall, Upper Saddle River, NJ, USA. 663pp.

Recebido em 01/09/2004

Aceito em 11/05/2006 